\title{
Discrete games of chance as models for continuous stochastic transport processes
}

\author{
Andrew Allison ${ }^{a}$ and Derek Abbott ${ }^{a}$ \\ ${ }^{a}$ Centre for Biomedical Engineering (CBME) and School of Electrical and Electronic \\ Engineering, University of Adelaide, SA 5005, Australia.
}

\begin{abstract}
Discrete games of chance can be used as to illustrate principles of stochastic processes. For example, most readers be familiar with the use of discrete random walks to model the microscopic phenomenon of Brownian motion. We show that discrete games of chance, such as those of Parrondo and Astumian, can be used to quantitatively model stochastic transport processes.

Discrete games can be used as "toy" models for pedagogic purposes but they can be much more than "toys." In principle we could perform accurate simulations and we could reduce the errors of approximation to any desired level, provided that we were prepared to pay the computational cost.

We consider some different approaches to discrete games, in the literature, and we use partial differential equations to model the particle densities inside a Brownian Ratchet. We apply a finite difference approach and obtain finite difference equations, which are equivalent to the games of Parrondo. The new games generalise Parrondo's original games, in the context of stochastic transport problems.

We provide a practical method for constructing sets of discrete games, which can be used to simulate stochastic transport processes. We also attempt to place discrete games, such as those of Parrondo and Astumian, on a more sound philosophical basis.
\end{abstract}

Keywords: Brownian ratchet, diffusive transport, distributed charge, Poisson's equation, Fokker-Planck equation

\section{INTRODUCTION}

The correct explanation of the problem of Brownian motion was first offered by Einstein ${ }^{1}$ in 1905 . Since that time, kinetic theory has been applied to ever larger and more complex molecules, including those that govern life, such as proteins and enzymes. ${ }^{2-6}$ Transport processes in nature are believed to involve both diffusion (caused by Brownian motion) and direct response to applied fields. These fields vary in time which prevents the processes from reaching thermodynamic equilibrium. One aspect of this non-equilibrium behaviour is a steady state flow of particles, in response to variations in the applied field. Devices of this type are called Brownian ratchets. $^{7-11}$ These devices have been extensively analysed and simulated and some researchers have been able to build functional devices based on the ratchet principle. ${ }^{12-15}$

Parrondo et al. have shown that the important transport properties of Brownian ratchets can be demonstrated using discrete games of change ${ }^{16-21}$ It is possible to construct parlour games, analogous to "craps" or "snakes and ladders," which demonstrate a counter-intuitive effect. It is possible to combine two "losing" games to achieve a "winning" result. Other authors, including Astumian, ${ }^{22}$ have proposed similar discrete games. Parrondo's games have proved to be a fertile ground for research. ${ }^{23-28}$ The applications often extend far beyond the original inspiration, of stochastic transport.

The use of discrete games to model stochastic processes has a long history. One well known example is the use of the toss of a coin to decide the direction of a random walk. ${ }^{29}$ It is possible to model processes of this type as discrete Markov chains. ${ }^{29,30}$ Parrondo's games clearly serve a pedagogical purpose as simplified or "toy"

Further author information: (Send correspondence to A. Allison, School of Electrical and Electronic Engineering, University of Adelaide, SA 5005, Australia.) E-mail: aallison@eleceng.adelaide.edu.au, Telephone:+61 883035283

Noise in Complex Systems and Stochastic Dynamics, Lutz Schimansky-Geier, Derek Abbott, Alexander Neiman, Christian Van den Broeck, Editors, Proceedings of SPIE Vol. 5114 (2003) 
models of complex natural phenomena. In this regard, they are similar to the random walk models, but the similarity does not end there. Just as discrete random tosses of a coin can be used to model continuous Brownian motion $^{30}$; we can use discrete random events to model other continuous stochastic processes, such as Brownian ratchets. In this paper, we argue that Parrondo's can be made much more realistic and quantitative and that the errors of approximation can be reduced to arbitrarily small values.

Harmer and Taylor et al. have shown that the theory of Markov chains ${ }^{29,31}$ can be applied to Parrondo's games ${ }^{16}$ in much the same way that it applies to random walks. Parrondo's games are a Markov process but they are not homogeneous in time. In the general case, it is possible that Parrondo's games may not approach equilibrium as $t \rightarrow \infty$ and the limiting probability distribution may not be well defined. Fortunately, it is often possible to re-define what is meant by "state" and in many cases the concepts of equilibrium and limiting probability distribution can be preserved, and used. ${ }^{25}$

A number of authors have considered the relationship between discrete and continuous ratchets. ${ }^{32-36}$ The two themes that run though this literature are the philosophical justification of the use of discrete games to model continuous processes and the need to numerically evaluate the master equations which are generally too difficult to solve analytically. We present an approach to this problem based on the method of finite differences.

\section{THE FOKKER-PLANCK EQUATION}

We seek a macroscopic statistical description for the diffusion of a very small particle in a uniform fluid, under the influence of external forces. There are two common approaches to this type of problem; to use a Stochastic Differential Equation (SDE) ${ }^{37}$, such as the Langevin equation, or to use a Partial Differential Equation (PDE), such as the Fokker-Planck Equation. ${ }^{38}$ Each approach has its advantages and disadvantages; its partisans and detractors. Kurtz ${ }^{39,40}$ has shown that is possible to directly approximate the solution to an SDE using a Markov chain. This is certainly a valid approach to the problem and is worthy of future consideration. We have found it more convenient to work with the Fokker-Planck PDE. Further discussion of the relative merits of the two approaches, SDE versus PDE, can be found in Risken. ${ }^{38}$

The dynamics of a flashing Brownian ratchet can be described using a partial differential equation called the Fokker-Planck equation, ${ }^{38}$ which describes the probability density, of finding a particle at a certain place and time, under the influence of diffusion and externally applied fields. We apply standard finite-difference methods of numerical analysis ${ }^{41}$ to the Fokker-Planck equation and derive a set of finite difference equations which have the same form as Parrondo's games.

We denote the probability of finding a Brownian particle at a certain point on space, $z$, and time, $t$, by $p=p(z, t)$. The time-evolution of $p(z, t)$ is governed by a partial differential equation called the Fokker-Planck equation:

$$
\frac{\partial^{2}}{\partial z^{2}}(D(z, t) p(z, t))-\frac{\partial}{\partial z}(\alpha(z, t) p(z, t))-\frac{\partial}{\partial t} p(z, t)=0
$$

The functions $\alpha(z, t)$ and $D(z, t)$ are referred to as the infinitesimal first and second moments of diffusion. In practice, the infinitesimal second moment does sometimes depend on concentration of the solute, $p(z, t)$, but is usually regarded as constant and is called the "Fick's law constant." A typical value (for a hydrated Sodium ion in water) would be of the order $D \approx 1.3 \times 10^{-9} \mathrm{~m}^{2} \mathrm{~s}^{-1}$. The infinitesimal first moment depends on the magnitude of externally imposed forces and on the mobility of the Brownian particle which is given by

$$
u=\frac{Z_{e}}{6 \pi \eta a}
$$

where $Z_{e}$ is the electrical charge on the particle, $\eta$ is the kinematic viscosity of the solvent and $a$ is the effective radius of the particle. A typical value for the mobility (of a hydrated Sodium ion in water) would be $u \approx$ $51.9 \times 10^{-9} \mathrm{~m}^{2} \mathrm{~s}^{-1}$ volt $^{-1}$. Further descriptions and numerical data may be found in books on physical chemistry and statistical physics. ${ }^{42-45}$ If we apply an electrical potential, or voltage, of $V(z, t)$ then the infinitesimal first moment is given by

$$
\alpha(z, t)=-u \frac{\partial}{\partial t} V(z, t)
$$


The theory behind Equations 2 and 3 is due to Stokes and Einstein. ${ }^{1}$ More information about the methods of solution and the applications of the Fokker-Planck equation can be found in Risken. ${ }^{38}$

When we take into account the functional forms of $D$ and $\alpha$ then we can rewrite the Fokker-Planck equation as:

$$
D \frac{\partial^{2} p}{\partial z^{2}}-\frac{\partial \alpha}{\partial z} p-\alpha \frac{\partial p}{\partial z}-\frac{\partial p}{\partial t}=0
$$

This is the form of the Fokker-Plank equation which we will sample at regular intervals in time and space, to yield finite difference equations.

\section{FINITE DIFFERENCE APPROXIMATION}

Many Partial Differential Equations, or PDEs, including Equation 4, can be very difficult to solve analytically. One well established approach to this problem is to sample possible solutions to a PDE at regular intervals, called mesh points. ${ }^{41}$ The true solution is approximated locally by a collocating polynomial. The values of the derivatives of the true solution are approximated by the corresponding derivatives of the collocating polynomial.

We can define local coordinates, expanded locally about a point $\left(z_{0}, t_{0}\right)$ we can map points between a real space $(z, t)$ and an integer or discrete space $(i, j)$. Time, $t$, and position, $z$, are modelled by real numbers, $t, z \in \mathcal{R}$ and the corresponding sampled position, $i$, and sampled time, $j$, are modelled by integers $i, j \in \mathcal{Z}$. We sample the space using a simple linear relationship

$$
(z, t)=\left(z_{0}+i \lambda, t_{0}+j \tau\right)
$$

where $\lambda$ is the sampling length and $\tau$ is the sampling time.

In order to map Equation 4 into discrete) space, we need to make suitable finite difference approximations to the partial derivatives. The notation is greatly simplified if we define a family of difference operators:

$$
\Delta_{i, j}=p\left(z_{0}+i \lambda, t_{0}+j \tau\right)-p\left(z_{0}, t_{0}\right)
$$

In principle, this is a doubly infinite family of operators but in practice we only use a small finite subset of these operators. This is determined by our choice of sampling points. This choice is not unique and is not trivial. The set of sampling points is called a "computational molecule. ${ }^{41}$ " Some choices lead to over-determined sets of equation with no solution. Some other choices lead to under-determined sets of equations with infinitely many solutions. We chose a computational molecule called "Explicit" computation with the following sample points: $(i, j) \in\{(0,0),(-1,-1),(0,-1),(+1,-1)\}$.

We also need to make a choice regarding the form of the local collocating polynomial. This is not unique and inappropriate choices do not lead to unique solutions. A polynomial which is quadratic in $z$ and linear in $t$ is the simplest feasible choice:

$$
p(z, t)=p\left(z_{0}, t_{0}\right)+A_{1} \cdot\left(z-z_{0}\right)+A_{2} \cdot\left(z-z_{0}\right)^{2}+B_{1} \cdot\left(t-t_{0}\right)
$$

where $A_{1}, A_{2}$ and $B_{1}$ are the real coefficients of the polynomial. Equations 5,6 and 7 imply a simple system of linear equations that can be expressed in matrix form:

$$
\left[\begin{array}{ccc}
-\lambda & +\lambda^{2} & -\tau \\
0 & 0 & -\tau \\
+\lambda & +\lambda^{2} & -\tau
\end{array}\right]\left[\begin{array}{c}
A_{1} \\
A_{2} \\
B_{1}
\end{array}\right]=\left[\begin{array}{c}
\Delta_{-1,-1} \\
\Delta_{0,-1} \\
\Delta_{+1,-1}
\end{array}\right] .
$$

These can be solved algebraically, using Cramer's method to obtain expressions for $A_{1}, A_{2}$ and $B_{1}$ :

$$
A_{1}=\frac{p\left(z_{0}+\lambda, t_{0}-\tau\right)-p\left(z_{0}-\lambda, t_{0}-\tau\right)}{2 \lambda}
$$

and

$$
A_{2}=\frac{p\left(z_{0}-\lambda, t_{0}-\tau\right)-2 p\left(z_{0}, t_{0}-\tau\right)+p\left(z_{0}+\lambda, t_{0}-\tau\right)}{2 \lambda^{2}}
$$


and

$$
B_{1}=\frac{p\left(z_{0}, t_{0}\right)-p\left(z_{0}, t_{0}-\tau\right)}{\tau}
$$

These are all intuitively reasonable approximations but their choice is not arbitrary. Equations $9,10,11$ form a complete and consistent set. We could not change one without adjusting the others. We can evaluate the derivatives of Equation 7 to obtain a complete and consistent set of finite difference approximations for the partial derivatives:

$$
\frac{\partial p}{\partial z}=A_{1}=\frac{p\left(z_{0}+\lambda, t_{0}-\tau\right)-p\left(z_{0}-\lambda, t_{0}-\tau\right)}{2 \lambda}
$$

and

$$
\frac{\partial^{2} p}{\partial z^{2}}=2 A_{2}=\frac{p\left(z_{0}-\lambda, t_{0}-\tau\right)-2 p\left(z_{0}, t_{0}-\tau\right)+p\left(z_{0}+\lambda, t_{0}-\tau\right)}{\lambda^{2}}
$$

and

$$
\frac{\partial p}{\partial t}=B_{1}=\frac{p\left(z_{0}, t_{0}\right)-p\left(z_{0}, t_{0}-\tau\right)}{\tau} .
$$

We can apply the same procedure to $\alpha(z, t)$ to obtain

$$
\frac{\partial \alpha}{\partial z}=A_{1}=\frac{\alpha\left(z_{0}+\lambda, t_{0}-\tau\right)-\alpha\left(z_{0}-\lambda, t_{0}-\tau\right)}{2 \lambda} .
$$

Equations 12, 13, 14 and 15 can be substituted into Equation 4 to yield the required finite partial difference equation:

$$
p\left(z_{0}, t_{0}\right)=a_{-1} \cdot p\left(z_{0}-\lambda, t_{0}-\tau\right)+a_{0} \cdot p\left(z_{0}, t_{0}-\tau\right)+a_{+1} \cdot p\left(z_{0}+\lambda, t_{0}-\tau\right)
$$

where

$$
a_{-1}=\frac{\frac{D \tau}{\lambda^{2}}+\frac{\alpha\left(z_{0}, t_{0}\right) \tau}{2 \lambda}}{\frac{\alpha\left(z_{0}+\lambda, t_{0}-\tau\right)-\alpha\left(z_{0}-\lambda, t_{0}-\tau\right)}{2 \lambda} \tau+1}
$$

and

$$
a_{0}=\frac{-2 \frac{D \tau}{\lambda^{2}}+1}{\frac{\alpha\left(z_{0}+\lambda, t_{0}-\tau\right)-\alpha\left(z_{0}-\lambda, t_{0}-\tau\right)}{2 \lambda} \tau+1}
$$

and

$$
a_{+1}=\frac{\frac{D \tau}{\lambda^{2}}-\frac{\alpha\left(z_{0}, t_{0}\right) \tau}{2 \lambda}}{\frac{\alpha\left(z_{0}+\lambda, t_{0}-\tau\right)-\alpha\left(z_{0}-\lambda, t_{0}-\tau\right)}{2 \lambda} \tau+1} .
$$

We can overload the arguments of $p$ and write them in terms of the discrete space $(i, j)$ using the mapping defined in Equation 5.

$$
p_{i, j}=a_{-1} \cdot p_{i-1, j-1}+a_{0} \cdot p_{i, j-1}+a_{+1} \cdot p_{i+1, j-1} .
$$

The meaning of the arguments should be clear from the context and from the use of subscript notation, $p_{i, j}$, rather than function notation, $p(z, t)$. Equation 20 is precisely the form required for Parrondo's games.

\section{PARRONDO'S GAMES}

In the original formulation, the conditional probabilities of winning or losing depend on the state, $i$, of capital but not on any other information about the past history of the games:

- Game $\mathrm{A}$ is a toss of a biased coin:

$$
p_{w i n}=\frac{1}{2}-\epsilon
$$

where $\epsilon$ is an adverse external bias that the game has to "overcome". This bias, $\epsilon$, is typically a small number such as $\epsilon=1 / 200$, for example. ${ }^{17,23}$

- Game B depends on the capital, $i$ : 
If $(i \bmod 3)=0$, then the odds are unfavourable.

$$
p_{\text {win }}=\frac{1}{10}-\epsilon
$$

If $(i \bmod 3) \neq 0$, then the odds are favourable.

$$
p_{w i n}=\frac{3}{4}-\epsilon .
$$

It is straightforward to simulate a randomised sequence of these games on a computer using a very simple algorithm. ${ }^{26}$

\subsection{Parrondo's "Game A" as a partial difference equation}

We can write the requirements for Game A in the form of Equation 20.

$$
p_{i, j}=\left(\frac{1}{2}-\epsilon\right) \cdot p_{i-1, j-1}+0 \cdot p_{i, j-1}+\left(\frac{1}{2}+\epsilon\right) \cdot p_{i+1, j-1} .
$$

This implies a constraint that $a_{0}=0$ which implies that $D \tau / \lambda^{2}=1 / 2$ which defines the relative scales of $\lambda$ and $\tau$ so we can give it a special name:

$$
\beta=\frac{D \tau}{\lambda^{2}} .
$$

The constraints on $a_{-1}$ and $a_{+1}$ imply a value for Parrondo's " $\epsilon$ " parameter:

$$
\epsilon=\left\{\frac{\lambda}{4 D}\right\} \alpha\left(z_{0}, t_{0}\right)
$$

which can be related back to an externally imposed electric field, $E=-\partial V / \partial z$ using equations 2 and 3 :

$$
\epsilon=\left(\frac{\lambda}{4 D}\right)\left(\frac{Z_{e}}{6 \pi \eta a}\right)\left(-\frac{\partial V}{\partial t}\right) .
$$

The small bias, $\epsilon$, is proportional to the applied external field which justifies Parrondo's original intuition.

\subsection{Parrondo's "Game B" as a partial difference equation}

There is still zero probability of remaining in the same state which implies a constraint that $a_{0}=0$ which implies that we still have the same scale, $\beta=\frac{1}{2}$. If we are in state $i$ then we can denote the probability of winning by $q_{i}=P($ win|initial position is $i)$. We can write the difference equations for game $\mathrm{B}$ in the form:

$$
p_{i, j}=q_{i-1} \cdot p_{i-1, j-1}+0 \cdot p_{i, j-1}+\left(1-q_{i+1}\right) \cdot p_{i+1, j-1} .
$$

which, together with Equations 17, 18 and 19, gives

$$
\frac{q_{i-1}}{1-q_{i+1}}=\frac{a_{-1}}{a_{+1}}=\frac{1+\frac{\lambda}{2 D \tau} \alpha_{i, j}}{1-\frac{\lambda}{2 D \tau} \alpha_{i, j}}
$$

which implies that

$$
\alpha_{i, j}=2 \lambda \beta \frac{q_{i-1}-\left(1-q_{i+1}\right)}{q_{i-1}+\left(1-q_{i+1}\right)} .
$$

This can be combined with Equation 3 and then directly integrated to calculate the required voltage profile. We can approximate the integral with a Riemann sum:

$$
V_{i}=-\frac{2 \beta}{u} \sum_{k=0}^{i} \frac{1-\left(\frac{1-q_{k+1}}{q_{k-1}}\right)}{1+\left(\frac{1-q_{k+1}}{q_{k-1}}\right)}
$$


so we can construct the required voltage profile for the ratchet which means that, given the values of $q_{i}$, it is possible to construct a physical Brownian ratchet that has a finite difference approximation which is identical with Parrondo's games. We can conclude that Parrondo's games are literally a finite element model of a flashing Brownian ratchet.

We note that Game B, as defined here, is quite general and actually includes Game A as a special case.

\subsection{Conditions for convergence of the solution}

There is a theorem due to O'Brien et al. ${ }^{46}$ which establishes that the numerical integration of a parabolic PDE, in explicit form, will converge to the correct solution as $\lambda \rightarrow 0$ and $\tau \rightarrow 0$ provided $\beta=D \tau / \lambda^{2} \leq \frac{1}{2}$. Under these conditions, the solution to the finite partial difference equation 20 would converge to the true solution of the partial differential equation 4 , as the mesh size, $\lambda$ went to zero. We see that Parrondo's choice of diffusion operator, with $\beta=\frac{1}{2}$ is at the very edge of the stable region.

\subsection{An appropriate choice of scale}

There is a feasible range of values for $\beta$ As $\beta \rightarrow 0$ we require the time step $\tau \rightarrow 0$ which means that the number of time steps required to simulate a given time interval, $T$, increases without bound $N_{\text {steps }}=T / \tau \rightarrow \infty$. It is computationally infeasible to perform simulations with very small values of $\beta$. On the other hand, the value of $\beta=1 / 2$ implied in Parrondo's games is at the very limit of stability. In fact, the presence of small roundoff errors in the arithmetic could cause the the discrete simulation to diverge significantly from the continuous solution.

We propose that choosing $\beta=1 / 4$, in the middle of the feasible range, is most appropriate. If we consider the case of pure diffusion, with $\alpha=0$, then Equation 20 reduces to

$$
p_{i, j}=\beta \cdot p_{i-1, j-1}+(1-2 \beta) \cdot p_{i, j-1}+\beta \cdot p_{i+1, j-1}
$$

and if we choose $\beta=1 / 4$ then this reduces to

$$
p_{i, j}=\frac{1 \cdot p_{i-1, j-1}+2 \cdot p_{i, j-1}+1 \cdot p_{i+1, j-1}}{4}
$$

which is the same as Pascal's triangle with every second row removed. The solution to where the initial condition is a Kronecker delta function, $p_{i, 0}=\delta_{i, 0}$ is easy to calculate:

$$
p_{i, j}=2^{-2 j} \cdot\left(\begin{array}{l}
2 j \\
j+i
\end{array}\right)=2^{-2 j} \cdot \frac{(2 j) !}{(j+i) !(j-i) !}
$$

which is a half period, or double frequency, binomial. We can invoke the Laplace and De Moivre form of the Central Limit Theorem which establishes a correspondence between Binomial, or Bernoulli, distribution and the Gaussian distribution to obtain

$$
p_{i, j}=\frac{1}{\sqrt{2 \pi\left(\frac{j}{2}\right)}} \exp \left(\frac{-(i)^{2}}{j}\right) .
$$

This expression is only approximate but is true in the limiting case as $j \rightarrow \infty$.

In the case where $\alpha=0$; the Fokker Planck Equation 4 reduces to a diffusion equation:

$$
D \frac{\partial^{2} p}{\partial z^{2}}-\frac{\partial p}{\partial t}=0
$$

Einstein's solution to the diffusion equation is a Gaussian probability density function:

$$
p(z, t)=\frac{1}{\sigma \sqrt{2 \pi}} \exp \left(\frac{-(z)^{2}}{2 \sigma^{2}}\right)
$$

where the variance, $\sigma^{2}$, is a linear function of time:

$$
\sigma^{2}=2 D t
$$


It is possible to verify that this is a solution by direct substitution:

$$
\begin{aligned}
D \frac{\partial^{2} p}{\partial z^{2}} & =\frac{\partial p}{\partial t} \\
& =\left(\frac{1}{2 t}\right) \cdot\left(\left(\frac{z}{\sigma}\right)^{2}-1\right) \cdot p(z, t) .
\end{aligned}
$$

If we sample this solution using the mapping in Equation 5 then we obtain Equation 35 again. This is an exact result. We conclude that the choice of $\beta=1 / 2$ is very appropriate for the solution to the diffusion equation. We suggest that this would also be true for the Fokker-Planck equation, in the case where $\alpha$ is "small." The appropriate choice of $\beta$, given arbitrarily large, or rapidly varying, $\alpha$ is still an unsolved problem.

\subsection{An example of a simulation}

We simulated a physically reasonable ratchet with a moderately large modulo value, $M=8$. (The value for the original Parrondo's games was $M=3$.) We used the value of $\beta=1 / 4$. The simulation was based on a direct implementation of Equation 20 in Matlab. We chose a sampling time of $\tau=12 \mu$ s and a sampling distance of $\lambda \approx 0.25 \mu \mathrm{m}$. The result is shown in Figure 1, where we indicate how the expected position of a particle can move within a Brownian flashing ratchet during four cycles of the modulating field. We can see a steady drift of the mean position of the particle in response to the ratchet action. This simulation includes a total of 500 time

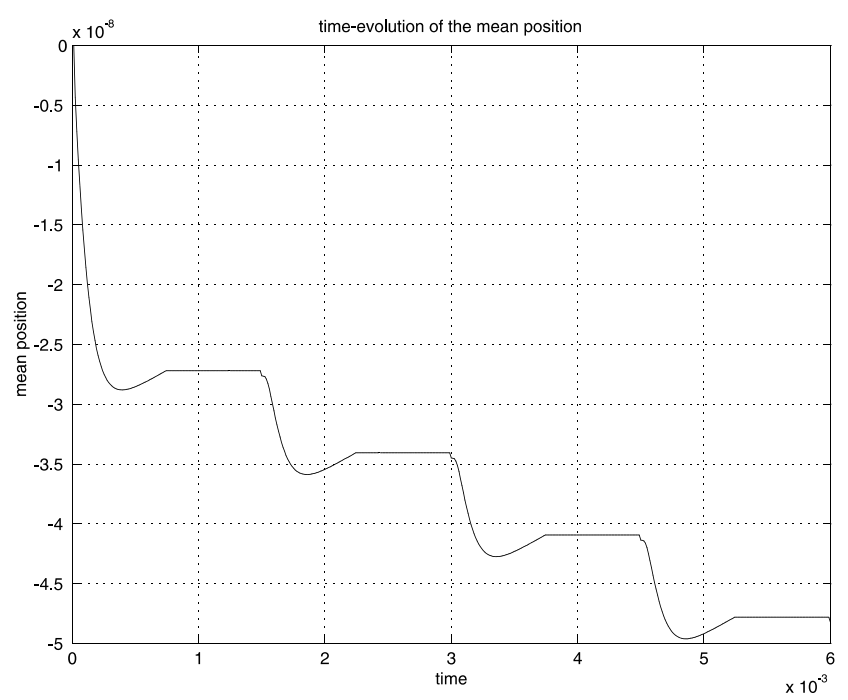

Figure 1. Time-evolution of the mean of the distribution $p(z, t)$. When the field is asserted, the mean position of the particles moves in a generally "downward" direction. There is some relaxation towards the end of that part of the cycle. When the field is turned off, the mean remains constant although diffusion causes the field to spread. The total shift in mean position of this ratchet is very modest, about $0.005 \mu \mathrm{m}$, compared with the spacing between the teeth of the ratchet, of $2.0 \mu \mathrm{m}$. Part of the motivation of this work is to optimise the transport effect of the Brownian ratchet, subject to constraints.

samples. Note that the average rate of transport quickly settles down to a steady value, even after only four cycles of the ratchet.

\section{CONCLUSIONS}

We conclude that Parrondo's games are a valid finite-difference simulation of a flashing Brownian ratchet.We affirm that Parrondo's " $\epsilon$ " parameter is a reasonable way to simulate a gradual externally imposed electric field, or voltage gradient. We have established that Parrondo's implied choice of the $\beta$ parameter does lead to a stable simulation but we suggest that the choice of $\beta=1 / 4$ is more appropriate from a mathematical point of view. 
Finally, we have generalised Parrondo's games, in the form of a set of finite difference equations 20 and we have shown that this can be implemented on a computer.

\section{REFERENCES}

1. A. Einstein, "On the movement of small particles suspended in a stationary liquid demanded by the molecular kinetic theory of heat," Ann. Physik. 17(17), p. 549, 1905. Reprinted in: Investigations on the Theory of the Brownian Movement, Dover, New York (1956).

2. H. Westerhoff, T. Y. Tsong, P. B. Chock, and R. D. Astumian, "How enzymes can capture and transmit free energy from an oscillating electric field," Proc. Natl. Acad. Sci. USA 83, pp. 4734-4738, July 1986.

3. R. D. Astumian, P. B. Chock, T. Y. Tsong, Y. C. Y., and H. Westerhoff, "Can free energy be transduced from electric noise?," Proc. Natl. Acad. Sci. USA 84, pp. 434-438, 1987. Brownian Ratchets.

4. R. D. Astumian, P. B. Chock, T. Y. Tsong, and H. Westerhoff, "Effects of oscillations and energy-driven fluctuations on the dynamics of enzyme catalysis and free-energy transduction," Phys. Rev. A. 39(12), pp. 6416-6435, 1989.

5. R. D. Astumian and M. Bier, "Fluctuation driven ratchets: Molecular motors," Physical Review Letters $\mathbf{7 2 ,}$ pp. 1766-1769, 1994.

6. D. Astumian, "Protein conformational fluctuations and free energy transduction," Appl. Phys. A 75, pp. 193-206, 2002.

7. C. R. Doering, "Randomly rattled ratchets," Il Nuovo Cimento 17D(7-8), pp. 685-697, 1995.

8. C. R. Doering, L. A. Dontcheva, and M. M. Klosek, "Constructive role of noise: Fast fluctuation asymptotics of transport in stochastic ratchets," Chaos 8(3), pp. 643-649, 1998.

9. B. Lindner, M. Kostur, and L. Schimansky-Gier, "Optimal diffusive transport in a tilted periodic potential," Fluctuation and Noise Letters 1(1), pp. R25-R39, 2001.

10. P. Reimann, "Brownian motors: noisy transport far from equilibrium," Physics Reports 361, pp. 57-265, 2002.

11. P. Reimann and P. Hänggi, "Introduction to the physics of Brownian motors," Appl. Phys. A. 75, pp. 169$178,2002$.

12. L. P. Faucheux, L. S. Bourdieu, P. D. Kaplan, and A. J. Libchaber, "Optical thermal ratchet," Phys. Rev. Lett. 74(9), pp. 1504-1507, 1995.

13. T. A. Duke and R. H. Austin, "Microfabricated sieve for the continuous sorting of macromolecules," Phys. Rev. Lett. 80(7), pp. 1552-1555, 1998.

14. D. Ertas, "Lateral separation of macromolecules and polyelectrolytes in microlithographic arrays," Phys. Rev. Lett. 80(7), pp. 1548-1551, 1998.

15. J. S. Bader, R. W. Hammond, S. A. Henk, M. W. Deem, G. A. McDermott, J. M. Bustillo, J. W. Simpson, G. T. Mulhern, and J. M. Rothberg, "Dna transport by a micromachined brownian ratchet device," PNAS 96(23), pp. 13165-13169, 1999.

16. G. P. Harmer, D. Abbott, P. G. Taylor, and J. M. R. Parrondo, "Parrondo's paradoxical games and the discrete brownian ratchet," in Abbott and Kish, ${ }^{47}$ pp. $149-160$.

17. G. P. Harmer, "Parrondo's paradox," Statistical Science 14, pp. 206-213, 1999.

18. G. P. Harmer and D. Abbott, "Losing strategies can win by Parrondo's paradox," Nature 402, p. 864, 1999.

19. C. E. M. Pearce, "Parrondo's paradoxical games," in Abbott and Kish, ${ }^{47}$ pp. 420-425.

20. C. E. M. Pearce, "Entropy, markov information sources and Parrondo's games," in Abbott and Kish, ${ }^{47}$ pp. $426-431$.

21. P. V. E. McClintock, "Parrondo's games and population genetics," Nature (London) 401, p. $23,1999$.

22. D. Astumian, "Making molecules into motors," Scientific American 285, pp. 56-64, July 2001.

23. G. P. Harmer, "The paradox of Parrondo's games," Proc. Royal Soc. ,Series A , (Math. Phys. and Eng. Science) 1994(99), pp. 247-259, 2000.

24. D. D. Meyer and H. Blumer, "Parrondo's games as lattice gas automata," in Parrondo's games as lattice gas automata, (Santa Fe, NM), 21-24 Aug. 2000. 
25. A. Allison and D. Abbott, "State-space visualisation and fractal properties of parrondo's games," in Proceedings of the Ninth International Symposium on Dynamic Games and Applications 2000, A. S. Nowak and K. Szajowski, eds., 1, pp. 1-6, The International Society of Dynamic Games (ISDG), December 2000. Submitted to Birkhauser Annals of the ISDG (in process) preprint: http://xxx.lanl.gov/abs/cond-mat/0205536.

26. G. P. Harmer, D. Abbott, P. G. Taylor, and J. M. R. Parrondo, "Brownian ratchets and parrondo's games," Chaos 11(3), pp. 1-10, 2001.

27. G. P. Harmer and D. Abbott, "A review of Parrondo's games," Fluctuation and Noise Letters 2(2), pp. R71R107, 2001

28. Y. Lee, A. Allison, D. Abbott, and H. E. Stanley, "A minimal Brownian ratchet: an exactly solvable model," Phys. Rev. Lett. , 2002. in process, preprint: http://xxx.lanl.gov/cond_mat/0205302.

29. H. M. Taylor and S. Karlin, An Introduction to Stochastic Modeling, Academic Press, San Diego, third ed., 1998.

30. M. Baxter and A. Rennie, Financial calculus: An Introduction to Derivative Pricing, Cambridge University Rress, Cambridge, 1996.

31. J. R. Norris, Markov Chains, Cambridge University Press, Cambridge, 1997.

32. L. Schimansky-Geier, M. Kschischo, and T. Fricke, "Flux of particles in sawtooth media," Phys. Rev. Lett. 79(18), pp. 3335-3338, 1997.

33. J. A. Freund and L. Schimansky-Geier, "Diffusion in discrete ratchets," Phys. Rev. E 60(2), pp. 1304-1309, 1999.

34. K. D. Heath, D. Kinderlehrer, "Discrete and continuous ratchets: From coin toss to molecular motor," Discrete and Continuous Dynamical Systems, Series B 2, pp. 153-167, May 2002.

35. A. Allison and D. Abbott, "The physical basis for Parrondo's games," Fluctuation and Noise Letters 2(4), pp. L327-L341, 2002. in process, preprint: http://xxx.lanl.gov/cond_mat/0208470.

36. R. Toral, P. Amengual, and S. Mangioni, "Parrondo's games as a discrete ratchet," Elsevier, 2003. Preprint only, in process.

37. B. Øksendal, Stochastic Differential Equations, Springer, Berlin, 1998.

38. H. Risken, The Fokker-Planck Equation, Springer, Berlin, 1985.

39. T. G. Kurtz, "Solutions of ordinary differential equations as limits of pure jump markov processes," J. Appl. Prob. 7, pp. 49-58, 1970.

40. T. G. Kurtz, "Limit theorems for sequences of jump markov processes approximating ordinary differential processes," J. Appl. Prob. 8, pp. 344-356, 1971.

41. L. Lapidus, Digital Computation for Chemical Engineers, McGraw-Hill Book Co. Inc., New York, 1962.

42. R. B. Bird, W. E. Stewart, and E. N. Lightfoot, Transport Phenomena, John Wiley \& Sons, New York, 1960.

43. F. Reif, Statistical Thermal Physics, McGraw-hill Book Co., Singapore, 1965.

44. P. W. Atkins, Physical Chemistry, Oxford University Press, Oxford, 1978.

45. E. L. Cussler, Diffusion Mass Transfer in Fluid Systems, Cambridge University Press, Cambridge, 1997.

46. G. G. O'Brien, M. A. Hyman, and S. Kaplan, “,” J. Math. Phys. 29, p. 223, 1951.

47. D. Abbott and L. B. Kish, eds., Unsolved problems of Noise and Fluctuations (UPoN'99), 511, American Institute of Physics (AIP), July 2000. 